\title{
BMJ Open Assessing care trajectories of adolescent females seeking early induced abortion in New South Wales: multistage, mixed- methods study protocol
}

\author{
Anisa Rojanapenkul Assifi (D) , ${ }^{1}$ Melissa Kang (D) , ${ }^{1}$ Elizabeth Sullivan (D) , \\ Angela J Dawson (i) ${ }^{1}$
}

To cite: Assifi AR, Kang M, Sullivan E, et al. Assessing care trajectories of adolescent females seeking early induced abortion in New South Wales: multistage, mixed-methods study protocol. BMJ Open 2020;10:e039819. doi:10.1136/ bmjopen-2020-039819

- Prepublication history for this paper is available online. To view these files, please visit the journal online (http://dx.doi. org/10.1136/bmjopen-2020039819).

Received 28 April 2020 Revised 02 October 2020 Accepted 06 October 2020

\section{Check for updates}

(c) Author(s) (or their employer(s)) 2020. Re-use permitted under CC BY-NC. No commercial re-use. See rights and permissions. Published by BMJ.

${ }^{1}$ School of Public Health, Faculty of Health, University of Technology Sydney, Sydney, New South Wales, Australia ${ }^{2}$ Office of the PVC Health and Medicine, Faculty of Health and Medicine, The University of Newcastle, Callaghan, New South Wales, Australia

Correspondence to Anisa Rojanapenkul Assifi; anisamra@gmail.com

\section{ABSTRACT}

Introduction In Australia, New South Wales (NSW), abortion has recently been removed from the criminal code. Previous research from Australia and other highincome countries has focused on adult women's access to abortion services. This protocol describes a five-stage mixed-methods study to determine the care trajectories and experiences of adolescent females, aged 16-19 years, seeking an early induced abortion in NSW. The aims are to (1) explore the needs and perspectives of adolescent females seeking sexual and reproductive health services in NSW and (2) develop a framework for abortion service provision for adolescents in NSW.

Methods and analysis This study comprises: (1) semistructured qualitative interviews with key informants, individuals with diverse, in-depth experience of providing and/or supporting abortion care in NSW; (2) a crosssectional online survey of adolescent females residing in NSW; (3) case study interviews with adolescents females who have accessed an abortion service in NSW; (4) a codesign workshop with adolescents who took part in stage 3 to develop relevant knowledge and recommendations and (5) a knowledge dissemination forum with key stakeholders.

Ethics and dissemination Ethics approval has been received from the University of Technology Sydney Human Research Ethics Committee for this study. Data collection commenced in March 2019 and will continue until the end of 2020. This study aims to develop a deep understanding of adolescent abortion care trajectories and experiences of abortion services in NSW. The study will deliver coproduced recommendations to improve adolescent access to abortion information and services.

\section{INTRODUCTION}

Every woman has the right to safe and highquality abortion services where they are treated with dignity and respect. ${ }^{1}$ In Australia and other high-income countries, access to abortion services can be limited by a lack of services (particularly in rural areas), cost and negative attitudes of staff. ${ }^{2-6}$ Locating medical abortion providers among primary care
Strengths and limitations of this study

- This method acknowledges and values adolescents expertise alongside that of health professionals and health service managers.

- The age range of adolescent participants is narrow and does not reflect the full adolescent experience.

- Adolescents surveyed will not be representative of all adolescents in New South Wales.

- Engaging adolescents in a co-design process will ensure the development of a framework for adolescent-centred quality abortion care and standards.

- A knowledge exchange forum will enable the framework to be disseminated to key decision makers and to advocate for adolescent access.

doctors may also be hampered by the legal context and provider concerns about stigma. ${ }^{7}$

Adolescents encounter more barriers than adults when attempting to access health services for non-stigmatised health issues; ${ }^{8}$ however, there is limited research that specifically examines barriers to abortion care for adolescents (defined by the World Health Organization (WHO as 10-19 years). Given the stigma associated with adolescent sexual behaviour, ${ }^{9}$ it seems likely that abortionrelated stigma ${ }^{10}$ may be intensified in this age group. Previous research in high-income countries has focused on adolescent decisionmaking and adolescent-restrictive abortion legislation. However, there is a minimal literature about adolescent experiences of abortion services and the provision of adolescent-friendly abortion services. ${ }^{11} 12$ The sensitive nature of adolescents and abortion, as well as the stigma associated with adolescent sexual behaviour may contribute to the lack of evidence concerning adolescent access to abortion care. 


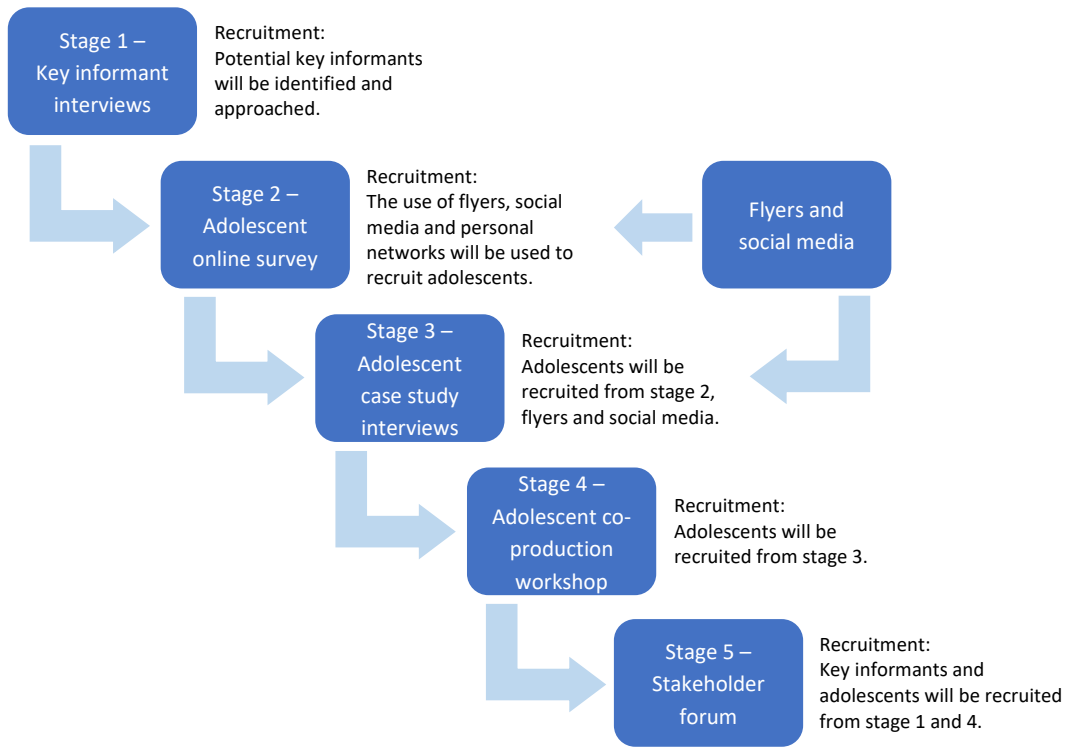

Figure 1 Diagram of study stages.

The state of New South Wales (NSW) removed abortion from the criminal code in September 2019. There are no mandated abortion services in the public health system. The current environment is, therefore, timely to deliver evidence to inform service delivery planning and improve access to abortion services to ensure that they meet the needs of the adolescent population.

In this study we use the term 'adolescent/s', 'adolescent female/s' and 'young women' when referring to our study participants. However, we realise that some study participants may not identify as 'female'. We acknowledge that not everyone who has a uterus or has had/may have an abortion identifies as female. The experience of unintended pregnancy is not limited to cis-gender, heterosexual women. Transmasculine people (assigned female at birth) and sexual minority women (bisexual or lesbian) are more likely to report experiences of mistimed or unwanted pregnancy than heterosexual women with only male partners and experience. ${ }^{13} 14$

This protocol describes a five-stage sequential exploratory mixed-methods study (figure 1), to determine the care trajectories and experiences of adolescent females, aged 16-19 years, seeking an early induced abortion in NSW.

\section{METHODS AND ANALYSIS}

The aims of this study are to:

1. Explore the needs and perspectives of adolescent females seeking sexual and reproductive health services in NSW with a particular focus on abortion services.

2. Develop a framework for abortion service provision for adolescents in NSW.

\section{Methodological approaches}

This study will adopt appreciative inquiry (AI) and co-production methodologies. AI is a strength-based, participatory approach that seeks to identify the positive characteristics, rather than the problems of an organisation and involves the members of that organisation in quality improvement. ${ }^{15-18}$ Co-production acknowledges that improvements to an organisation or health system delivery must be compelled by both the health professionals/stakeholders as well as the individuals using the service. ${ }^{19}$ This approach does not seek to diminish the expertise of health professionals and stakeholders but acknowledges and champions that consumers such as adolescents also come with their own level of expertise. ${ }^{20}$ In our study, adolescent women and other stakeholders are included in the development of study method and 
design, through the Research Advisory Group. Adolescents will participate in the development of framework and recommendations. This approach will facilitate the engagement of key stakeholders and the dissemination of information about the study and its findings. The study is aligned with the goals and expected outcomes of the 2017 NSW Youth Health Framework 2017-2024, concerning the provision of quality healthcare. This involves building support mechanisms to increase access to services, improve the adolescent healthcare experience and engage adolescents to contribute to the design of service pathways and information to enable appropriate health decision-making. ${ }^{21}$

The study will comprise five stages; Stages 1-3 will explore the needs, experiences and perspectives of adolescents and other stakeholders. Stage 4 will use synthesised findings from the first three stages to develop a framework for service provision. The final stage will involve a stakeholder forum to present the framework and gather feedback.

\section{Stakeholder engagement}

A Research Advisory Group was established in late 2018 to provide input into the research methods, ethical considerations, piloting of tools of the research study and recruitment of key informants through their networks. The group includes the research team, adolescent and young adult women (18-22 years) and health professionals with expertise in abortion care in NSW. Through the Research Advisory Group and stages of the study, there is direct engagement with individuals who have expert knowledge of the abortion field in NSW.

Young women have been involved in the development of the study and design, and piloting of all research tools as members of the Research Advisory Group. In the workshop in stage 4 of the study, adolescents will 'co-produce' a framework and recommendations with the research team to guide the future provision of adolescent-friendly abortion services. Young people will also assist in presenting the framework to stakeholders for feedback in the dissemination stage of the study. Some of these adolescents and young people will include those who have experienced abortion care in NSW. This research seeks to empower adolescents to ensure their voice determines how services should be provided to them.

\section{Patient and public involvement}

Adolescent and healthcare professionals were involved in the development of the study design, study tools and recruitment. This has been described in the previous sections.

\section{Stage 1}

Aim

To explore the experiences and views of key informants concerning abortion care, access and service navigation in NSW.
Design

Cross-sectional, qualitative study using one-on-one semistructured interviews.

\section{Participants}

'Key informants' in our study are health service managers and clinicians (general practitioners, gynaecologists and practice nurses) who have in-depth knowledge and experience of abortion care in NSW.

\section{Recruitment and sample size}

Potential participants were identified through the research team and Research Advisory Group and invited by email. A second email was sent after 2 weeks, followed by either another email or a telephone call after a further 2 weeks. Sample size was 5-10 participants from diverse professional backgrounds, organisations and geographic locations. Given the limited number of abortion services in NSW, we anticipated that this would provide sufficient breadth and depth of data required. The sample size is small as we purposively selected participants based on their expert insight and knowledge. ${ }^{22}$

\section{Data collection}

Semistructured interviews were conducted face-to- face or via telephone between February 2019 and December 2019. Interviews lasted $30-45 \mathrm{~min}$ each, were audio recorded and transcribed verbatim. An interview schedule was developed in consultation with the Research Advisory Group (see figure 2) and piloting of the interview tool was conducted in January 2019. The questions, taking an AI approach, explored participant perspectives of abortion service delivery in NSW to adolescents, access pathways and opinions about, and suggestions for, service and system strengths and improvements.

\section{Analysis}

Data analysis is currently underway (August 2020). A directed content analysis will be employed ${ }^{23}$ using two lenses to understand both the environment of abortion services and to seek insights into strategies to improve care. A framework for abortion access, developed from the findings of a study in NSW that explored women's access to abortion, ${ }^{24}$ will be used in the first part of the analysis. This ecological model outlines four overarching domains that impact on and determine quality abortion services. These domains seek to describe factors that affect abortion service provision and uptake that are embedded in the: structures of society (eg, legal, political and education institutions), health system, community and those that may be in line with the values, preferences and needs of women themselves. The framework will

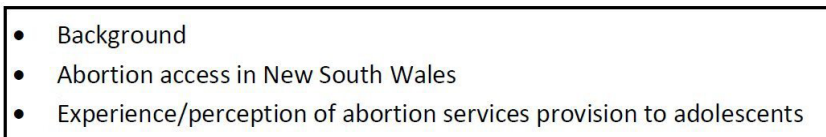

Figure 2 Stage 1 interview schedule headings. 
guide the coding of such that emergent themes will be mapped to these four domains. The key themes will then be understood in terms of the four phases of AI: discover, dream, design and deliver. This will enable the identification of positive policy and practice that could be built on in future services as well as provide insights into how abortion services should be implemented and evaluated. The analysis will be conducted by three researchers to achieve inter-rater reliability. The software package NVivo V.1 $12^{25}$ will support the data organisation and analysis. The Consolidated Criteria for Reporting Qualitative Research (COREQ) guideline will be followed in the reporting of results. ${ }^{26}$

\section{Expected outcomes}

An in-depth understanding of abortion service provision for adolescents in the current NSW health context and recommendations for service and system improvements.

\section{Consent}

Written consent was obtained from all participants.

\section{Stage 2}

Aim

To explore adolescent women's access to, and sources of information on sexual and reproductive health and their experience of sexual health and abortion service provision.

\section{Design}

Cross-sectional online survey.

\section{Participants}

Adolescent females aged 16-19 years residing in NSW. Although WHO defines adolescents as those aged 10-19 years, ${ }^{27}$ we will restrict the age range for this study in consideration of age of consent and mandatory reporting legislation in NSW. Given the sensitive nature of the study, this will minimise risk to participants. This age range also reflects the average age at which adolescent females in Australia become sexually active, which is 16 years. ${ }^{28}$

\section{Recruitment and sample size}

Participants were recruited online and offline. Online recruitment occurred through: (1) paid, targeted social media advertising through Facebook and Instagram; (2) posting and sharing on Facebook and Twitter through relevant social networks, such as student organisations and collectives and (3) advertising on partner organisations social media pages. The Research Advisory Group and researcher networks identified relevant organisations and services who distributed flyers. We used chain-referral sampling ${ }^{29}$ whereby, adolescents who completed the survey were asked to share the link to the questionnaire with their peers and other individuals in their network. The online survey targeted females aged 16-19 years, a range of terminology was used in recruitment materials including 'young women', 'teenagers' and '16-19year olds'.

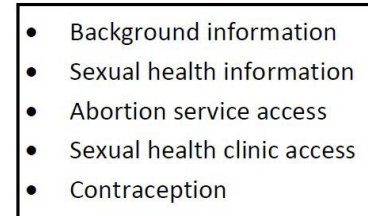

Figure 3 Stage 2 questionnaire topic headings.

Different URLs were provided for each of the recruitment methods (Facebook, Instagram, emails, snowballing) to allow us to track which methods were achieving the greatest uptake. This allowed us to shift resources to focus recruitment using the most effective methods.

At the end of the online survey, participants were invited to enter a draw to receive one of five $\$ 30$ gift vouchers.

To report frequencies with a $95 \%$ CI, our target sample size was 384, based on 184859 females aged 16-19 in NSW in 2018. ${ }^{30}$ The formula from Krejcie and Morgan's paper was used to calculate a representative sample size. ${ }^{31}$

\section{Data collection}

The survey was administered through SurveyGizmo. ${ }^{32}$ Survey questions were developed from published research ${ }^{3433}$ that explored adolescent sexual reproductive health, youth friendly health service and abortion service access (figure 3). Demographic information sought included age in years, gender, postcode of residence, country of birth, language spoken at home and level of education. Questions about sources of information for sexual and reproductive development and health topics including contraception and abortion, and their scope, were included. Abortion service utilisation was asked, and for respondents who answered in the affirmative, further questions about their trajectory, timeline and experience of those services were included. Participants were asked whether they had sought care for sexual and reproductive health reasons. Confirmatory responses lead to further questions about their experience of care in those services. The survey was piloted with adolescent members of the Research Advisory Group and their peers. It took approximately $15 \mathrm{~min}$ to complete. Most questions were a combination of multiple-choice questions, Likert Scale questions and open-text questions.

\section{Data analysis}

Data analysis is currently underway (August 2020). Data were exported directly from SurveyGizmo into the statistical software programme SPSS V.25. ${ }^{34}$ We will describe frequencies and analyse associations between demographic and all other variables using $\mathrm{X}^{2}$ tests. Alpha will be 0.05 .

Qualitative content analysis will be used to analyse freetext responses to questions about abortion trajectories, experiences and timelines. We will use conventional coding methods, deriving themes directly from the text data.

\section{Expected outcomes}

To quantify and describe adolescent self-reported experiences of access to sexual and reproductive healthcare and abortion knowledge and services in NSW. 
Consent

Consent was obtained by inviting the participant to scroll through the 'participant information' and select the statement 'I consent to take part in the survey' before entering the survey.

\section{Stage 3}

Aim

To explore the experiences of adolescents who have accessed abortion services in NSW.

\section{Design}

Case study, using one-on-one semistructured interviews.

\section{Participants}

Adolescent and young adult females, aged 16-24 years, who have accessed an abortion service in NSW between the ages of 16 and 19, and within 5 years.

\section{Recruitment}

Participants will be recruited through the online survey and offline. At the end of the survey (Stage 2), participants who indicated that they accessed an abortion service will be asked if they are interested in taking part in an interview. Potential participants will opt-in; a phone number and email address will be provided at the end of the survey and interested participants invited to make contact. Once contacted the researcher will provide further information about the interview process and assess eligibility for the study. Offline recruitment will occur by contacting partner organisations, non-governmental organisation health services and abortion providers across NSW to display and handout recruitment flyers. We aim to continue recruitment of adolescents and young women with diverse experiences of abortion to obtain an in-depth understanding of their unique contexts. We anticipate interviewing approximately 5-10 adolescents. This sample size is in line with other qualitative studies in the area of adolescent abortion. ${ }^{35-37}$

\section{Data collection}

Data collection commenced in late 2019 and will conclude by December 2020. One-on-one semistructured interviews will be conducted face-to-face or by telephone. Faceto-face interviews will take place in a convenient location agreed on between the participant and interviewer where privacy can be maintained. Interviews will take between 60 and $90 \mathrm{~min}$. All interviews will be audio recorded and then transcribed verbatim. An interview schedule has been developed, with input provided by the Research Advisory Group and piloted with two youth representatives. The interview guide was developed based on the AI approach, the questions focused on the positive aspects of adolescent's experience and care trajectories. The guide includes questions concerning decision-making, how information was accessed, expectations going into a service, experiences of care, and suggestions for service strengths and improvements (see figure 4).

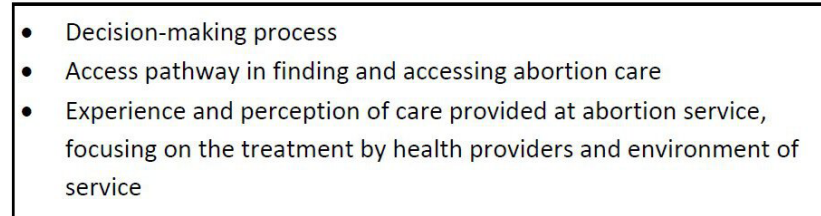

Figure 4 Stage 3 interview schedule headings.

\section{Data analysis}

Interview transcripts will form the data for thematic analysis. The analysis will be informed by grounded theory and will use Glaser and Strauss' approach of 'constant comparison'. 38 Data will be entered into and coded inductively using the software package NVivo, V.12. ${ }^{25}$ Coding will be guided by identifying the strengths and strategies that were employed by participants to overcome barriers and address needs. The 'constant comparison' approach will be used to find consistencies and differences in order to refine the concepts and themes. The emergent themes will be discussed among three researchers to achieve inter-rater reliability. By examining the codes across and within all transcripts, the final themes will be determined by consensus. ${ }^{39}$ Concept mapping will then be used to elaborate each adolescent's 'story'. Exploring abortion access and experience from the point of view of the study participants. The final themes will be mapped to the concept map to clarify how they relate to each other. Reporting of results will follow the COREQ guidelines. ${ }^{26}$

\section{Expected outcomes}

To gain an in-depth understanding of adolescent experience and care trajectories when accessing abortion services in NSW. We will also gain insight into how abortion service provision should be and could be provided from adolescents' perspective.

\section{Consent}

Written consent will be obtained from all participants before the commencement of the interview.

\section{Stage 4}

Aim

To develop a framework for abortion service provision for adolescents in NSW.

\section{Design}

Qualitative participatory action research involving a half-day, facilitated, co-design workshop will be conducted with adolescents and service providers. Participatory action research emphasises a collaborative approach, requiring adolescents and service providers to participate in developing relevant knowledge. ${ }^{41}$

\section{Participants}

Adolescent females aged 16-24 years who have accessed an abortion service in NSW when aged 16-19 years, and service providers. 


\section{Recruitment}

Stage 3 participants will be invited to take part in the workshop. The sample size will be six adolescents and service providers.

\section{Data collection}

A half-day workshop will take place in a meeting room with the participants and the research team. Researchers will take notes throughout the workshop. The workshop will involve the following processes:

1. Mind mapping ${ }^{42}$

The mind mapping process will be used to develop an ideal adolescent abortion service framework. Adolescent participants and service providers will conduct a mind map to describe optimal abortion service delivery and access, and to identify those elements which are already occurring and those which are not.

2. Affinity diagram

All participants will then develop an affinity diagram ${ }^{43}$ based on information from stages 1-3 to formulate recommendations for adolescent abortion care. An affinity diagram is used to bring together information and arrange the different ideas, opinions and issues into categories and subcategories. These will form the recommendations. The following steps will be taken to develop the affinity diagram:

- Participants will brainstorm how adolescent abortion service delivery should and could look like. Adolescents will generate ideas and opinions based on their personal experience. They will write each idea or opinion on a single sticky notes. Identified key points from stages 1 and 2 of the study will also be written on sticky notes.

- The sticky notes with the ideas will be put up around the room to display them.

- The participants and researchers will be invited to read the different ideas and discuss whether they agree and disagree with the idea. Once everyone has read the ideas the participants will be asked to sort the ideas into related groups. Participants and the researchers will keep sorting until all sticky notes are placed in groups.

- Participants will examine the sticky notes in each group to identify an idea that conveys the theme of that group and can serve as the header. If no sticky note does, participants will come up with a header that defines the group. The names of the headers will be reached through consensus.

3. Finalise recommendations and framework.

The participants will review and revise the adolescent abortion service framework developed through the Mind mapping exercise until consensus is reached. The final agreed on framework will be recorded by the researchers. Participants will finalise the recommendations developed through the affinity diagram. Participants will review, revise and clarify the recommendations and groupings. Researchers will then document the final recommendations.

\section{Expected outcomes}

A framework and recommendations to inform adolescentfriendly abortion service delivery in NSW.

Consent

Written consent will be obtained from all participants.

\section{Synthesis and interpretation of data}

In stage 4 of the study, data from all stages will be synthesised to enhance understanding and ensure the framework incorporates all perspectives. This final stage of data analysis involves the integration of the separate phases of quantitative and qualitative results (inferences) into coherent and meaningful 'meta-inferences'. To achieve this, a 'triangulation protocol' ${ }^{44}$ will be used to converge the data. ${ }^{45}$ A matrix will be developed, displaying all the emerged themes and findings from each stage of the study. The themes and findings will then be reviewed by the researchers to determine whether there is 'agreement', 'partial agreement', 'silence' or 'dissonance' between the stages. 'Silence' is where a theme has only arisen from a single stage. Through this process 'metathemes' will be identified.

\section{Stage 5: stakeholder forum}

A knowledge forum will be held after the study. Participants from stages 1 and 4 of the study will be invited and the co-designed framework and recommendations for the provision of adolescent abortion will be presented by participants from stage 4 . Meta-themes identified through the integration and interpretation of data will also be presented. A structured workshop format will be used to seek specific feedback to refine the framework.

\section{STUDY TIMELINE}

Participant recruitment and data collection in stages 1 and 2 commenced in early 2019 and were completed in December 2019. Stage 3 commenced in late 2019 and stages 4 and 5 have not yet commenced.

\section{ETHICS AND DISSEMINATION}

Ethical approval was received in December 2018 from the University of Technology Sydney Human Research Ethics Committee (Approval number:ETH18-2216) for stages 1-4. As we will be asking adolescent females to recall their personal experiences accessing an abortion, they may potentially experience emotional distress. To minimise this potential risk, a distress protocol outlining how support and counselling services will be provided has been developed to guide the research team.

Findings from the study will also be disseminated via peer reviewed journal publications and conference presentations. 
There are some limitations of this study. First, by excluding younger adolescents we will not be able to understand the needs of a particularly vulnerable group. This younger group of adolescents is underrepresented in the research. ${ }^{46}$ Second, there may be recruitment bias of adolescents into the quantitative online survey due to our sampling strategy. The survey sample is not representative of adolescents living in NSW as convenience, chain-referral and targeted sampling is used to recruit participants. However, these methods may facilitate recruitment of a hard-to-reach population group and allow enquiry into what might be perceived as a sensitive subject. ${ }^{29}$ Through chain-referral sampling we are targeting multiple different networks, sites, which expands the scope beyond a single network. Increasing the ability to reach this, at times, isolated population group. $^{29}$

Young women's participation in co-producing a framework for adolescent abortion service provision provides opportunity for female empowerment and acknowledges the value of adolescent knowledge, views and experiences. The co-production and dissemination of a framework for adolescent-centred abortion care services in NSW, will assist to advocate for adolescent-centred quality abortion care and standards that could be transferrable to other states and territories in Australia and beyond.

Twitter Anisa Rojanapenkul Assifi @anisaassifi, Melissa Kang @DrMelissaKang and Angela J Dawson @AngelaJDawson

Acknowledgements We would like to thank our Research Advisory Group and youth consultants for their active engagements, feedback and support for this project.

Contributors ARA is the lead researcher and wrote the draft for the protocol manuscript. All authors contributed to the study design and provided feedback on the manuscript. All authors read and approved the final manuscript.

Funding This research received internal university funds from the University of Technology Sydney (Higher Degree Research Development Award Round 3, 2018 \#041218-172590).

Competing interests None declared.

Patient and public involvement Patients and/or the public were involved in the design, or conduct, or reporting, or dissemination plans of this research. Refer to the Methods and analysis section for further details.

Patient consent for publication Not required.

Provenance and peer review Not commissioned; externally peer reviewed.

Open access This is an open access article distributed in accordance with the Creative Commons Attribution Non Commercial (CC BY-NC 4.0) license, which permits others to distribute, remix, adapt, build upon this work non-commercially, and license their derivative works on different terms, provided the original work is properly cited, appropriate credit is given, any changes made indicated, and the use is non-commercial. See: http://creativecommons.org/licenses/by-nc/4.0/.

\section{ORCID iDs}

Anisa Rojanapenkul Assifi http://orcid.org/0000-0001-5295-4074

Melissa Kang http://orcid.org/0000-0002-9438-2518

Elizabeth Sullivan http://orcid.org/0000-0002-8718-2753

Angela J Dawson http://orcid.org/0000-0003-0926-2202

\section{REFERENCES}

1 World Health Organization. Safe abortion: technical and policy guidance for health systems. World Health Organization, 2012.
2 Doran F, Nancarrow S. Barriers and facilitators of access to firsttrimester abortion services for women in the developed world: a systematic review. J Fam Plann Reprod Health Care 2015;41:170-80.

3 Finer LB, Frohwirth LF, Dauphinee LA, et al. Timing of steps and reasons for delays in obtaining abortions in the United States. Contraception 2006;74:334-44.

4 Nickson C, Smith AMA, Shelley JM. Travel undertaken by women accessing private Victorian pregnancy termination services. Aust NZ J Public Health 2006;30:329-33.

5 Silva M, Ashton T, McNeill R. Improving termination of pregnancy services in New Zealand. N Z Med J 2011;124:83-90.

6 Silva M, McNeill R, Ashton T. Factors affecting delays in first trimester pregnancy termination services in New Zealand. Aust $N Z \mathrm{~J}$ Public Health 2011;35:140-5.

7 Dawson AJ, Nicolls R, Bateson D, et al. Medical termination of pregnancy in general practice in Australia: a descriptive-interpretive qualitative study. Reprod Health 2017;14:39.

8 Hargreaves DS, Greaves F, Levay C, et al. Comparison of health care experience and access between young and older adults in 11 highincome countries. J Adolesc Health 2015;57:413-20.

9 Patton GC, Sawyer SM, Santelli JS, et al. Our future: a lancet commission on adolescent health and wellbeing. Lancet 2016;387:2423-78.

10 Hanschmidt F, Linde K, Hilbert A, et al. Abortion stigma: a systematic review. Perspect Sex Reprod Health 2016;48:169-77.

11 Assifi A, Kang M, Sullivan EA, et al. Abortion service provision for adolescents in high-income countries: a qualitative synthesis of the evidence 2019.

12 Ely GE, Hales TW, Jackson DL, et al. Access to choice: examining differences between adolescent and adult abortion fund service recipients. Health Soc Care Community 2018;26:695-704.

13 Everett BG, McCabe KF, Hughes TL. Sexual orientation disparities in mistimed and unwanted pregnancy among adult women. Perspect Sex Reprod Health 2017;49:157-65.

14 Agénor M, Cottrill AA, Kay E, et al. Contraceptive beliefs, decision making and care experiences among transmasculine young adults: a qualitative analysis. Perspect Sex Reprod Health 2020;52:7-14.

15 Koster RLP, Lemelin RH. Appreciative inquiry and rural tourism: a case study from Canada. Tourism Geographies 2009;11:256-69.

16 Reed K. Antenatal screening and the gendering of genetic responsibility. Reprod Health 2007;4:8.

17 Trajkovski S, Schmied V, Vickers M, et al. Using appreciative inquiry to transform health care. Contemp Nurse 2013;45:95-100.

18 Zandee DP. Appreciative inquiry and research methodology. In: Coghlan D, Brydon-Miller M, eds. The SAGE encyclopedia of action research. London: SAGE Publications Ltd, 2014: 49-51.

19 Alakeson V, Bunnin A, Miller C. Coproduction of health and wellbeing outcomes: the new paradigm for effective health and social care. London: OPM Group, 2013.

20 Batalden M, Batalden P, Margolis P, et al. Coproduction of healthcare services. BMJ Quality \&Safety 2015;0:1-9.

21 NSW Health. NSW youth health framework 2017-24. New South Wales: NSW Government, 2017.

22 Malterud K, Siersma VD, Guassora AD. Sample size in qualitative interview studies: guided by information power. Qual Health Res 2016;26:1753-60.

23 Hsieh H-F, Shannon SE. Three approaches to qualitative content analysis. Qual Health Res 2005;15:1277-88.

24 Family Planning New South Wales. Access to early abortion in New South Wales: Health provider and women's perspectives EXECUTIVE SUMMARY of a qualitative research study for Family Planning New South Wales, June 2016. Sydney, 2016.

25 NVivo. NVivo qualitative data analysis software Version 12 [program], 2018.

26 Tong A, Sainsbury P, Craig J. Consolidated criteria for reporting qualitative research (COREQ): a 32-item checklist for interviews and focus groups. Inter J Qual Health Care 2007;19:349-57.

27 World Health Organization. Adolescent health, 2018World Health Organization. Available: http://www.who.int/topics/adolescent_ health/en/ [Accessed 11 February 2018].

28 Fisher CM, Waling A, Kerr L, et al. 6th national survey of Australian secondary students and sexual health 2018. Bundoora: Australian Research Centre in Sex, Health \& Society, La Trobe University, 2019.

29 Penrod J, Preston DB, Cain RE, et al. A discussion of chain referral as a method of sampling hard-to-reach populations. J Trans Nurs 2003;14:100-7.

30 Australian Bureau of Statistics. Estimated resident population by age and sex at 30 June 2018 Canberra: Commonwealth of Australia, 2019. Available: https://www.abs.gov.au/AUSSTATS/abs@.nsf/ DetailsPage/3101.0Dec\%202018?OpenDocument [Accessed 22 Jun 2019]. 
31 Krejcie RV, Morgan DW. Determining sample size for research activities. Educ Psychol Meas 1970;30:607-10.

32 Widgix LLC dba SurveyGizmo. SurveyGizmo. Boulder, CO, 2019. www.surveygizmo.com

33 Baroudi M, Waenerlund A-K, San Sebastian M, et al. Assessing the dimensionality of YFHS-Swe: a questionnaire to assess youthfriendliness in differentiated health services. Glob Health Action 2017; $10: 1380399$

34 IBM. IBM SPSS Statistics for Windows Version 25.0 [program]. Armonk, NY: IBM Corp, 2017.

35 Andrews J, Boyle J. African American adolescents' experiences with unplanned pregnancy and elective abortion. Health Care Women Inter 2003;24:414-33.

36 Deeb-Sossa N, Billings DL. Barriers to abortion facing Mexican immigrants in North Carolina: choosing folk healers versus standard medical options. Latino Studies 2014;12:399-423.

37 Halldén B-M, Christensson K, Olsson P. Meanings of being pregnant and having decided on abortion: young Swedish women's experiences. Health Care Women Int 2005;26:788-806.

38 Glaser B, Strauss A. The discovery of grounded theory: strategies for qualitative research. New York: Routledge, 1967.
39 Braun V, Clarke V. Using thematic analysis in psychology. Qual Res Psychol 2006;3:77-101.

40 Ayres L. Thematic coding and analysis. In: Given L, ed. The SAGE encyclopedia of qualitative research methods. Thousand Oaks, California: SAGE Publications, 2008.

41 Pant M. Participatory Action Research. In: Coghlan D, Brydon-Miller $\mathrm{M}$, eds. The SAGE encyclopedia of action research. London: SAGE Publications Ltd, 2014: 583-8.

42 Burgess-Allen J, Owen-Smith V. Using mind mapping techniques for rapid qualitative data analysis in public participation processes. Health Expect 2010;13:406-15.

43 Sahay A. The seven new tools for quality improvement. Data visualization, Volume II : uncovering the hidden pattern in data using basic and new quality tools. New York: Business Expert Press LLC, 2017.

44 O'Cathain A, Murphy E, Nicholl J. Three techniques for integrating data in mixed methods studies. BMJ 2010;341:c4587.

45 Onwuegbuzie AJ, Combs JP. Emergent data analysis techniques in mixed methods. Thousand Oaks: Sage, 2010: 397-430.

46 Igras SM, Macieira M, Murphy E, et al. Investing in very young adolescents' sexual and reproductive health. Glob Public Health 2014;9:555-69 